\title{
The Value of Commission on Cancer Accreditation: Improving Survival Outcomes by Enhancing Compliance with Quality Measures
}

\author{
Subhasis Misra, MD, MS, FACCWS, FACS ${ }^{1}$, Ji Fan, MD, MBA ${ }^{1}$, Ujwal Yanala, MD $^{2}$, and \\ Chandrakanth Are, MD, MBA $^{2}$ \\ ${ }^{1}$ Surgical Oncology, Brandon Regional Hospital, HCA West FL Division/USF Health Consortium, Brandon, FL; ${ }^{2}$ Division \\ of Surgical Oncology, Department of Surgery, University of Nebraska Medical Center, Omaha, NE
}

In the United States, colorectal cancer is the third leading cause of cancer-related deaths in men and women and the second combined leading cause of cancer deaths. ${ }^{1}$ Nodal staging accuracy for colorectal carcinoma is one of the most important determinant for prognosis and the selection of patients for adjuvant chemotherapy. ${ }^{2}$ The American College of Pathologists in its consensus statement in 1999 defined accurate nodal staging to be a Category 1 prognostic factor and recommended that all identified lymph nodes be sectioned and a minimum of 12 lymph nodes be examined to accurately identify the American Joint Committee on Cancer (AJCC) stage III colon cancer. ${ }^{3}$ The AJCC 5th edition recommended obtaining at least 12 lymph nodes in radical colon resection, whereas AJCC 6th edition modified the recommendation to obtain at least 7-14 lymph nodes. ${ }^{4,5}$ The AJCC 7th edition modified this again to obtain at least 10-14 lymph nodes in radical colorectal resection. ${ }^{6}$ The AJCC 8 has not changed the $\mathrm{N}$ staging but clarified prognostic values of isolated tumor cells in lymph nodes (N0) and micrometastases $(\mathrm{N} 1){ }^{7}$ In its up-to-date guideline version 4.2018, the National Comprehensive Cancer Network (NCCN) has maintained that examination of a minimum of 12 lymph nodes must be one of the principles of colon cancer surgery. ${ }^{8}$ Failing to retrieve 12 lymph nodes in resection specimens is an indication for

(C) Society of Surgical Oncology 2019

First Received: 6 March 2019;

Published Online: 29 March 2019

S. Misra, MD, MS, FACCWS, FACS

e-mail: Subhasis.Misra@hcahealthcare.com consideration of adjuvant chemotherapy in stage II colon cancer. ${ }^{8}$ Therefore, the efforts to achieve the recommended benchmark has the potential to decrease patient's unnecessary exposure to the toxicity of adjuvant treatment. ${ }^{9}$

The American College of Surgeons (ACS) Commission on Cancer $(\mathrm{CoC})$ has identified this standard (12 Regional Lymph Nodes: "12 RLN") as a quality performance indicator for surgical treatment and this has also been endorsed by the National Quality Forum (NQFNQF\#0225) from October 30, 2018. The CoC has utilized this quality improvement measure (Standard 4.5) to assess performance at the hospital or systems-level but not at individual physician level. The CoC expects the program's performance rate to be equal to or greater than the Expected Performance Rate (EPR) specified for " 12 RLN," which is $85 \% .^{10,11}$

In this issue of the Annals of Surgical Oncology, Dr. Shulman et al queried approximately $1500 \mathrm{CoC}$ cancer programs to determine rates of compliance with the " 12 RLN" quality measure from 2003 to 2015 . The overall compliance improved from 52.8 to $92.1 \%$ during the study period and across all types of hospitals with compliant cases having better risk-adjusted survival than noncompliant cases. ${ }^{12}$ Although differences remain between the various $\mathrm{CoC}$ programs, the absolute compliance gap between the NCI designated, academic centers, and the community hospitals has significantly reduced from 25 to $6.4 \%$. The authors also noted that left sided tumors and community hospitals had lower compliance rates for " 12 RLN." This could be due to the technical challenges involved in mobilizing the left colon and the tendency for some to perform limited segmental resection. 
The association of increased lymph node recovery with increased survival may represent an effect of higher lymph node yields itself or may be a marker for other related factors, such as the quality of surgical resection or more accurate staging leading to delivery of more appropriate cancer-directed treatment. ${ }^{13}$ The improvement of compliance of "12RLN" may partially contribute to overall improvement of survival for colon cancer over the study period.

Accurate staging requires an appropriate operation and a concerted pathologic effort to identify lymph nodes in the colon specimen. ${ }^{14}$ The surgeon and pathologist are both responsible for compliance with the "12RLN" metric. The surgeon is required to remove and submit colon cancer specimen en bloc with adequate proximal and distal margins and inclusion of the regional mesenteric lymphatics following anatomical landmarks. The adequacy of 12 lymph node retrieval would increase from $60 \%$ to $90 \%$ if the average length of resected bowel increases from 25-30 to $36-42 \mathrm{~cm} .{ }^{15}$ Tattooing of colonic lesions at the time of preoperative colonoscopy also increases the quality of lymph node analysis. ${ }^{16}$ If fewer than 12 nodes are identified, additional visual enhancement techniques should be considered. ${ }^{3}$ Methylene blue staining, modified Davidson's fixation, or fat dissolution enables to detect more small lymph nodes and micrometastases in the resected colon and mesentery. ${ }^{17-19}$

In addition, the number of lymph nodes may vary from person to person. Interestingly, as in this paper, Asian race/ Pacific Island descent (presumably less obese than other races), younger age, higher stage of colon cancer, and right-side colon cancer had more compliance with " 12 RLN," which may reflect the fact that more lymph nodes are harbored in the mesentery of these certain populations. $^{20,21}$ Shulman's data also endorses the previous finding that the number of lymph nodes evaluated after surgical resection confers survival benefits for patients with stage II and III colon cancer. ${ }^{21,22}$ However, for stage III colon cancer, Tsikitis argued that the total number of lymph nodes analyzed is not a prognostic indicator of cancer-specific and disease-free survival. ${ }^{23}$ Vather further stated that the survival advantage appears to plateau beyond 16 lymph nodes, and the lymph node ratio seems to be a valid predictor of survival in Stage III cancer. ${ }^{21}$

The value of $\mathrm{CoC}$ accreditation is reflected in the analysis of this paper, and as noted even smaller community hospitals can have improved compliance upon adoption of these measures, and these will likely translate into subsequent increased survival. One also can make a case against regionalization in the care of certain cancers, provided that patients are treated in hospitals following accepted quality standards. Overall, quality metrics, such as lymph node count, albeit with some debate, have and will continue to elevate the standards of care for cancer patients.

\section{REFERENCES}

1. Sovich JL, Sartor Z, Misra S. Developments in screening tests and strategies for colorectal cancer. Biomed Res Int. 2015;2015:326728.

2. Natalie EJ, Sigurdson ER, Hanlon AL, Wang H, Mayer RJ, MacDonald JS, Catalano PJ, Haller DG. Accuracy of determining nodal negativity in colorectal cancer on the basis of the number of nodes retrieved on resection. Ann Surg Oncol. 2003;10(3):213-8.

3. Compton CC, Fielding LP, Burgart LJ, Conley B, Cooper HS, Hamilton SR, Hammond ME, Henson DE, Hutter RV, Nagle RB, Nielsen ML, Sargent DJ, Taylor CR, Welton M, Willett C. Prognostic factors in colorectal cancer. College of American Pathologists Consensus Statement 1999. Arch Pathol Lab Med. 2000;124(7):979-94.

4. AJCC 5th edition. https://cancerstaging.org/references-tools/des kreferences/Documents/AJCC5thEdCancerStagingManual.pdf. Accessed 26 Feb 2019.

5. AJCC 6th edition. https://cancerstaging.org/references-tools/des kreferences/Documents/AJCC6thEdCancerStagingManualPart1. pdf. Accessed 26 Feb 2019.

6. AJCC 7th edition. https://cancerstaging.org/references-tools/des kreferences/Documents/AJCC7thEdCancerStagingManual.pdf. Accessed 26 Feb 2019.

7. Weise MR. AJCC Colorectal Cancer, 8th edn. Ann Surg Oncol. 2018;25(6):1454-5.

8. NCCN guideline Colon Cancer, Version 2.2018. Accessed $26 \mathrm{Feb}$ 2019.

9. Earle CC, Weiser MR, Ter Veer A, Skibber JM, Wilson J, Rajput A, Wong YN, Benson AB 3rd, Shibata S, Romanus D, Niland J, Schrag D. Effect of lymph node retrieval rates on the utilization of adjuvant chemotherapy in stage II colon cancer. J Surg Oncol. 2009; 100(7):525-8.

10. CoC Quality of Care Measures. American College of Surgeons. Available at: https://www.facs.org/quality-programs/cancer/ncdb/ qualitymeasures. Accessed 7 Feb 2019.

11. National Quality Forum. http://www.qualityforum.org/Projects/ c-d/Cancer_Endorsement_Maintenance_2011/0225-Commis sion_on_Cancer,_ACS.aspx. Accessed 7 Feb 2019.

12. Shulman LN, Browner AE, Palis BE, et al. Compliance with cancer quality measures over time and their association with survival outcomes: the Commission on Cancer's Experience with the Quality Measure requiring at least 12 regional lymph nodes to be removed and analyzed with colon cancer resections. Ann Surg Oncol. 2019.

13. Siegel RL, Miller KD, Fedewa SA, et al. Colorectal cancer statistics, 2017. CA Cancer J Clin. 2017;67(3):177-93.

14. Compton CC. Key Issues in reporting common cancer specimens: problems in pathologic staging of colon cancer. Arch Pathol Lab Med. 2006;130(3):318-24.

15. Gravante G, Parker R, Elshaer M, et al. Lymph node retrieval for colorectal cancer: Estimation of the minimum resection length to achieve at least 12 lymph nodes for the pathological analysis. Int J Surg. 2016;25:153-7.

16. Dawson K, Wiebusch A, Thirlby RC. Preoperative tattooing and improved lymph node retrieval rates from colectomy specimens in patients with colorectal cancers. Arch Surg. 2010;145(9):826-30.

17. Reima H, Saar H, Innos K, Soplepmann J. Methylene blue intraarterial staining of resected colorectal cancer specimens improves 
accuracy of nodal staging: a randomized controlled trial. Eur J Surg Oncol. 2016;42(11):1642-6.

18. Kelder W, Inberg B, Plukker JT, et al. Effect of modified Davidson's fixative on examined number of lymph nodes and TNM-stage in colon carcinoma. Eur J Surg Oncol. 2008;34(5): $525-30$.

19. Maeda H, Okamoto K, Oba K, et al. Lymph node retrieval after dissolution of surrounding adipose tissue for pathological examination of colorectal cancer. Oncol Lett. 2018;15(2):2495-500.

20. Betge J, Harbaum L, Pollheimer MJ, et al. Lymph node retrieval in colorectal cancer: determining factors and prognostic significance. Int J Colorectal Dis. 2017;32(7):991-8.

21. Vather R, Sammour T, Kahokehr A, Connolly AB, Hill AG. Lymph node evaluation and long-term survival in Stage II and
Stage III colon cancer: a national study. Ann Surg Oncol. 2009;16(3):585-93.

22. Chang GJ, Rodriguez-Bigas MA, Skibber JM, Moyer VA. Lymph node evaluation and survival after curative resection of colon cancer: systematic review. J Natl Cancer Inst. 2007;99(6): 433-41.

23. Tsikitis VL, Larson DL, Wolff BG, Kennedy G, Diehl N, Qin R, Dozois EJ, Cima RR. Survival in stage III colon cancer is independent of the total number of lymph nodes retrieved. J Am Coll Surg. 2009;208(1):42-7.

Publisher's Note Springer Nature remains neutral with regard to jurisdictional claims in published maps and institutional affiliations. 